\title{
XIII.
}

\section{Erwiderung auf Körner's kritische Bemerkungen zu meiner Arbeit über die otitische Pyämie. \\ Von}

Dr. Ernst Lentert, Professor der Ohrenheilkunde in Giessen.

Im Ansehluss an das von Streit in der vorangehenden Arbeit über die irrige Auffassung Körner's betreffs der von mir angegebenen diagnostischen Bedeutung der Lumbalpunction Gesagte möchte ich auf einige andere Stellen in demselben Buche Kö rn er's (Otitische Erkrankungen u. s. w. 3. Aufl. 1902), in welchen er auf meine Pyämiearbeit Bezug nimmt, eingehen. Kö rner schreibt S. 98: „Zwar hat Leutert behauptet, meine nach seiner Versicherung, altersschwache“ Theorie habe keine Stütze, da ,noch keines Sterblichen Auge eine Phlebitis im kranken Warzenfortsatze gesehen habe. Sollen denn die im vereiternden Knochen verlaufenden Gefässchen vielleicht gesund bìeiben? Freilich muss Levtert zugeben, dass Zaufal Thrombose kleiner Knochenvenen an der Einmündungsstelle in den thrombosirten Sinus gesehen hat, fügt aber hinzu, dass hier, natürlich die Erklärung: mindestens eben so nahe liegt, dass sich die Thrombose vom Sinus auf die Venen fortgepflanzt hat'. $O b$ das wirklich so natïrlich ist, scheint mir von geringer Wichtigkeit, da anch Andere (Grunert und Zeroni, A. f. 0., XLIX, 185, Fall III) eine thrombosirte Vene im kranken Warzenfortsatze gefunden haben, die in den Sinus führte und mit Bestimmtheit annehmen, dass erst von dieser Vene aus der Sinus erkrankt war. Wachsen nun Thromben aus solchen Knochengefässen in den Sinus hinein, so kommt es natürlich zu einer wandständigen Thrombose, die nach Leutert das ron mir als Osteophlebitispyämie aufgefasste Krankheitsbild verursachen soll. Dass dies möglich ist, wird Niemand bestreiten, aber Leutert hat es nicht bewiesen, denn unter den 11 Fällen, die er seinen Erörterungen zu Grunde legt, ist nur einer (Fall V), der vielleicht zu meinem Krankheitsbilde der Osteophlebitispyämie gehört."s 
Die beiden ersten Sätze dieses Passus müssen bei dem Leser den Eindruck erwecken, als ob ich die Möglichkeit bestritten hätte, dass die kleinen Venen des Warzenfortsatzes bei Entzündung desselben thrombosiren könnten. Ich habe aber anf derselben Seite, auf welcher meine von Körner angeführten Worte stehen (S. 221) geschrieben: „Für ihre Fälle (Osteophlebitispyämie) hielten es die Meisten für das Wahrseheinlichste, dass die Thrombose durch Fortleitung ron den Knochenvenen her entstanden sei. Ich will diese Möglichkeit an sich ja nicht bestreiten, nur ist damit noeh lange nicht bewiesen, dass die Thrombose der Knochenvenen allein Pyämie hervorrufen kann." S. 226 führe ich dann weiter aus, weshalb Letzteres sogar unwahrscheinlich ist. Meine von Körner angeführten Worte dienten nur zur Erklärung des von mir gebrauchten Wortes "Theorie", was unzweifelhaft aus dem Zusammenhange hervorgeht, denn ich habe geschrieben: "denn eine Theorie ist es, da noeh keines Sterblichen Auge eine Phlebothrombose des Warzenfortsatzes gesehen hat". Die letzteren Worte halte ich auch

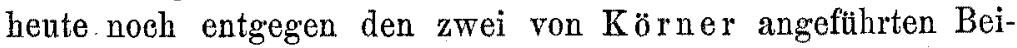
spielen (siehe die angeführten nächsten Sätze K örner's) aufrecht, da weder von Körner, dem es obgelegen hätte, noch von anderer Seite hinreichende Untersuchungen über Thrombose von Warzenfortsatzvenen bei Pyämie insonderheit bei dieser ohne Sinusthrombose vorliegen. Auch die von mir gegebene Erklärung betreffs $Z$ aufals Beobachtung von Thrombosen kleiner Venen an der Einmiundungsstelle in den Sinus halte ich heute noch für natürlich, da sich diese Thrombosen eben an der Einmündungsstelle in den Sinus fanden.

Ebenso unrichtig ist der Körner'sche Satz, dass nach Leutert die wandständige Thrombose das von ihm als Osteophlebitispyämie aufgefasste Krankheitsbild verursachen soll. Ich habe S. 235 geschrieben: „Beide Arten von Thromben, die wandständigen wie die obturirenden, können je nach ihrer Beschaffenheit and dem Stadium des Zerfalles beide Arten der allgemeinen Intoxication (Verschiedenartigkeit der Metastasen ist gemeint) hervorbringen. Ist die dem Lumen des Sinus zugekehrte Fläche glatt, so werden keine Thrombentheilchen in die Blutbahn übergeführt, desgleichen wenn der Zerfall eines Thrombus nicht an einer Stelle stattfindet, welche von der Circulation bertihrt wird, oder wenn die Zerfallsproducte rein eitrig-flüssiger Natur sind." Tch halte es dann weiterhin für wahrscheinlich, dass die 
wegen ihrer glatten Oberfläche wenig zum bröckeligen Zerfall neigende wandständige Thrombose besonders häufig den Pyämien nach acuter Ohreiterung zu Grunde liegt, weil ja jeder der hier in Frage kommenden Thromben zunächst als wandständiger entsteht, und der mit den virulenteren Entzündungserregern der acuten Eiterung inficirte Thrombus früher pyämische Erscheinungen machen wird, als der nach chroniseher Eiterung entstandene, weleher sich bis zum obturirenden entwickeln kann, ohne dass zunächst schwere Symptome auftreten. Hiermit gebe ich die Erklärung dafür, dass sich die Gelenk- und Muskelmetastasen, welche von nicht in Thrombenbröckeln eingehüllten Mikroorganismen erzeugt werden, besonders häufig bei Pyämien nach acuten Eiterungen finden, schliesse aber, wie aus den Eingangs angeführten Worten unzweifelhaft hervorgeht, keineswegs aus, dass diese Art der Metastasen allein auch bei obturirenden Thromben bezw. bei chronischer Eiterung vorkommen können. Es kommt eben einzig und allein darauf an, ob Eiter resp. Mikroorganismen . und Toxine allein, oder ob Thrombenbröckel in die Blutbahn gelangen; das Erstere ist aus den angeführten Gründen häufiger bei der wandständigen Thrombose (die auch bei der chronischen Eiterung vorkommt), das Letztere häufiger bei der obturirenden (die sich auch bei acuter Eiterung bilden kann). Aus dem nun folgenden Satze Körn er's ( ist....") könnte für diejenigen Leser, welche meine Arbeit nicht kennen, hervorgehen, dass ich die Absicht gehabt habe, einen derartigen Beweis zu liefern, dass mir dieses aber misslungen sei. Ich habe aber eine derartige Beweisführung gar nicht nöthig gehabt, daher auch gar nicht unternommen. Es genügte vollkommen, Körner die Möglichkeit vorzuhalten, dass sein Krankheitsbild auch bei Sinusthrombose vorkommen kann. Sobald diese Möglichkeit zugegeben ist, was Körner nunmehr gethan hat, so verliert sein Krankheitsbild jede Berechtigung, als ein besonderes auf einer besonderen anatomischen Unterlage ruhendes hervorgehoben zu werden. Es liegt eben im Wesen aller Theorien, dass ihnen das Gegentheil nicht bewiesen zu werden braucht, um sie zu stürzen, sondern es genügt der Nachweis, dass die der Theorie zu Grunde liegenden Beobachtungen anderer Deutungen fähig sind.

Nun glaube ich aber weiterhin, dass mein Fall V thatsächlich dem Körner'schen Krankheitsbilde entspricht, dass jedenfalls das Wort "vielleicht" hier zu wenig sagt. Körner muss 
zugeben, dass die bei Weitem grössere Wahrscheinlichkeit dafür spricht, dass die am Eingange berichteten Gelenkschwellungen als Metastasen aufzufassen sind. Es ist doch nichts Ungewöhnliches, dass sich eine Sinusthrombose entwickelt, ohne dass es zur Perforation des Trommelfelles kommt, resp. ohne dass die eventuell geringe Ohreiterung bemerkt wird. Ist es hier, wo es sich nicht um Scharlach handelt, nicht ganz unwahrscheinlich, dass eine Mittelohrentzündung, welche am 6 . November beginnt (Auftreten der ersten Schmerzen), bereits 13 Tage später zu einer so völligen Erweichung der Sinuswand geführt hat, dass sie am Punctionsstich von allein weiter einreisst? Weist nicht der Operationsbefund im Warzenfortsatz auf eine schon längere Zeit bestandene Mastoiditis hin? Und spricht das Sectionsprotokoll nicht ausdrücklich von einem ,älteren ausgedehnten, an mehreren Stellen eitrig zerfallenen Thrombus?" Es ist doch wohl kaum anzunehmen, dass ein so beschaffener Thrombus erst 9 Tage alt sein soll (rom Tage des ersten Wiederauftretens der Temperatursteigerung an gerechnet), sowie dass ein anseheinend so wenig acut einsetzender Process in 4 Tagen (Beginn der Schmerzen bis zum Wiederansteigen der Temperatur) zur Sinusthrombose fuhrt. Ist dieses aber alles unwahrscheinlich, so lassen sich auch die Gelenkschwellungen nur noch als Metastasen auffassen.

Auf S. 99 seines Buches macht Körner mir den Vorwurf, ich hätte übersehen, dass Schwartze schon vor Körner diejenige Annahme (Existenz der Osteophlebitispyämie) als zu Recht bestehend hingestellt habe, die ich nicht als die meines ehemaligen Chefs, sondern allein als die seinige auf das Heftigste bekämpfte. Wenn dieser Vorwurf berechtigt wäre, so hätte ich nicht allein Schwartze, sondern fast alle Autoren angreifen müssen, welche früher über otitische Pyämie berichtet haben. Die Anschauung, dass eine Pyämie ohne Sinusthrombose häufig allein vom Warzenfortsatz ausgehen könnte, war fitiher eben die allgemeine. In der letzten Zeit drängte indessen die Entwickelung unserer Disciplin immer mehr zu der Erkenntniss hin, dass den otitischen Pyämien so gut wie stets eine Thrombose eines Blutleiters zu Grunde liege. Dieser Entwickelung war die ältere Auffassung, welche gewissermaassen nur eine Ueberlieferung darstellte, nicht hinderlich, wohl aber die Ausführung K örner's, welcher den Versuch gemacht hatte, die ältere Anschanung systematisch zu stützen. Daher musste sich mein Angriff auch gegen ihn und nicht gegen die älteren Vertreter dieser Anschauung 
richten. Das Gleiche gilt von dem von Kö rn er S. 100 über die von mir angeblich vergessene Priorität der S chwartze'schen Schule in der Frage der Verbreitung septischer Stoffe auf dem Lymphwege (septische Verlaufsform) Gesagte.

S. 108 fragt $\mathrm{Körner}$ in Bezug auf die von mir angegebene Diagnosenstellung der Sinuserkrankung aus über mebrere Tage anhaltendem hohen Fieber: "1. Was sollen wir unter an hal te ndem Fieber verstehen? Nach Leu tert ist das Fieber anhaltend, wenn es ,über mehrere Tage dauert. Wieviel sind ,mehrere' Tage? Bei der auch von Leutert anerkannten häufigen Schwierigkeit, die Ursache eines Fiebers zu erkennen, genügen doch wohl mehrere Tage nicht, um jede andere Ursache für dasselbe auszuschliessen."

Hätte ich eine bestimmte Anzahl von Tagen angegeben, was schlechterdings unmöglich ist, so hätte ich allerdings dem Vorwurfe, in einen öden und den Kranken gefährdenden Schematismus verfallen zu sein (siehe S. 109), nicht entgehen können. Das "mehrere Tage" bedeutet hier nur, wie sich das jeder Einsichtige selbst sagen wird, eine Warnung davor, sofort beim ersten Eintreten hoher Temperaturen eine Sinusthrombose diagnosticiren zu wollen. Im Uebrigen habe ich aber gerade, weil die Diagnose aus hohem Fieber allein - Ohrerkrankung natürlich vorausgesetzt - zu leicht zu überflïssigen operativen Eingriffen verleiten kann, eine besondere Vorsichtsmaassregel angegeben. Ich rathe, in zweifelhaften Fällen zunächst den Sinus freizulegen, und falls dessen Aussehen keine Indication zur Eröffnung giebt, zunächst abzuwarten, ob das Fieber abfällt. Geschieht dieses bis zum Morgen des 3. Tages nicht (S. 240 meiner Arbeit), so halte ich die Diagnose Sinusthrombose für sicher. Es kann übrigens nur ganz ansnahmsweise vorkommen, dass Jemand nach "mehreren" Tagen eine vorhandene andere Ursache hohen Fiebers nicht entdeckt; und deshalb werden wir doch ein so werthvolles und einfaches diagnostisches Mittel in seiner Bedeutung nicht herabwürdigen.

Körner fährt dann fort: „2. Bei welchem Temperaturgrade sollen wir das Fieber als, hoch ${ }^{6}$ ansprechen? Le utert meint, bei $39^{\circ}$ und darüber. Dieser Grad ist aber zur Zeit noch ganz willkürlich angenommen."

Körner bezieht sich doch sonst so gern auf v. Bergmann, warum hat er den Hinweis unterlassen, dass dieser sich dieselbe "Willkürlichkeit" hat zu Schulden kommen lassen? v. Bergman $\mathrm{n}$ schreibt in der 3. Auflage seiner "Chirurgischen Behandlung von 
Hirnk rankheiten" S.581: "Am dringendsten sind die acuten Fälle. Wenn zu diesen (also denen einer acuten Otitis media) sich eine Eiterung im Warzenfortsatze und hohes Fiebers von $39^{\circ}$ und mehr gesellt hat und trotz Freiheit des Eiterabflusses aus dem aufgemeisselten Knochen nicht aufhört, so ist der Sinus freizulegen und näher zu untersuchen." v. B ergmann vertritt hier einen ganz ähnlichen Standpunkt, wie ich ihn, meines Wissens allerdings 3 Jahre früher, präcisirt habe. Ich glaube aber ein besonderes Recht dazu gehabt zu haben, gerade diesen Temperaturgrad als Grenze zu wählen, nachdem ich als Erster an der Hand einer grossen Anzahl von Warzenfortsatzerkrankungen und Epiduralabscessen mit genanen Temperaturmessungen, deren Publication allerdings erst 2 Jahre später erfolgt ist (in meinen Bacteriologisch-klinischen Studien u. s. w.), nachgewiesen habe, dass derartige Processe, solange der Sinus nicht mitergriffen bezw. dessen Wand nicht durchlässig geworden ist, niemals von hohen Temperaturen (die höchste betrug 38,7) begleitet sind. Wenn ich nun zur Sicherheit, und deswegen, weil $39^{\circ}$ auch allgemein pathologisch als Grenze zwischen mittleren und hohen Temperaturen angenommen wird, noch 3 Zehntel-Grade zugegeben habe, so kann man das doch nicht ohne Weiteres als "willkürlich angenommen" bezeichnen! Wo dasselbe vor mir von Körner, Jansen und Grunert nachgewiesen sein soll, habe ich nicht finden können. Körner hat nur von den cariösen Processen des Warzenfortsatzes bei chronischer Eiterung gesagt, dass bei ihnen Fieber nicht häufig sei; das ist doch etwas ganz Anderes! Auch bei Jansen fehlt die Verallgemeinerung, die hier allein von Werth ist, während die Grunert'sche Zusammenstellung, wie ich betont habe, hier überhaupt kaum herangezogen werden kann, da sie diesen Schluss nicht gezogen hat.

3. fragt Körner: "Wo liegt die entscheidende Grenze des Kindesalters? Leutert spricht von kleinen Kindern, bei denen die genannten $\mathrm{Ohr}$ - und Schläfenbeineiterungen auch ohne Sinusphlebitis mit hohen Temperaturen verlaufen können. Es kommt ja anch auf anderen Gebieten der Pathologie vor, dass ein und dieselbe Krankheit bei Kindern bisweilen mit stärkerer Ausprägung bestimmter Symptome, so auch des Fiebers, verläuft, als bei Erwachsenen, aber niemals sind diese verschiedenen Verlaufsformen der Krankheit dureh eine bestimmte Altersgrenze geschieden, vielmehr verwischt sich der Unterschied mit zunehmendem Alter allmählich, und die im 
Kindesalter häufigere Verlaufsform tritt vereinzelt auch noch bei jungen Leuten auf."

Was Körner hier gesperrt gedruckt gesagt hat, habe ich geglaubt, als allgemein bekannt voraussetzen zu dürfen. Ich habe es daher auch unterlassen, die entscheidende Grenze des Kindesalters auf Tag und Stunde festzusetzen, Körner hătte jedenfalls auch dann noch die Bestimmung der Minuten und Sekunden vermisst. Wenn er aber sagt, dass die im Kindesalter häufigere Verlaufsform vereinzelt auch noch bei jungen Leuten (wo liegt die entscheidende Altersgrenze?) auftritt, so habe ich zwar nicht nöthig, dieses abzulehnen, da es sich nur um Ausnahmefälle handelt, welche meiner Auffassung im Princip nicht widerstreiten, doch muss Körner für diese Behauptung in Bezug auf die Sinusthrombose Beweise beibringen; die Anführung von Beispielen genügt hier nicht. Wenn Körner von mir schreibt, dass bei kleinen Kindern die genannten Ohr- und Schläfenbeineiterungen auch ohne Sinusphlebitis mit hohen Temperaturen verlaufen können, so hat er mich nicht ganz richtig eitirt. Ich habe hier nur von aeuten Empyemen des Warzenfortsatzes, nicht aber von acuten Ohreiterungen gesprochen, bei welchen ja auch Erwachsene nicht selten hohes Fieber haben, und, was sehr wichtig ist und daher hier nicht weggelassen werden durfte, hinzugefügt, dass das Fieber, wenn es überhaupt vorhanden ist, nur kurze Zeit anzubalten pflegt. Der meinem betreffenden Satz vorausgehende (S. 227): „Bei kleinen Kindern liegt die Sache insofern anders, als sie erfahrungsgemäss schon auf die Aufnahme kleiner Mengen infectiosen Materiales mit hohem Fieber reagiren", ist absichtlich ganz allgemein gehalten. Hierbei ist beispielsweise und besonders an die vielleicht durch Verdauungsstörungen bervorgerufenen, bei sonst vollkommenem Wohlbefinden in den ersten 2 Lebensjahren häufiger auftretenden Temperatursteigerungen zu denken, welche zuweilen sehr hoch sind und mehrere Tage anhalten. Im Uebrigen muss es aber im Einzelfalle dem Arzt überlassen bleiben, zu entscheiden, ob das Fieber auf das $\mathrm{Ohr}$ zu beziehen ist, oder nicht. Aber ich trage Eulen nach Athen, auch Körner weiss ja ganz genau, was kleine Kinder sind, er schreibt gleich in dem dem angeführten folgenden Absatz: "Bei der acuten Paukenhöhlenentzündung, namentlich wenn dieselbe beide Ohren befällt, zeigen kleine Kinder häufig höhere 'Temperaturen, als Erwachsene..."

Auf S. 130 schreibt Körner: "Ueber die Stelle, an welcher Archiv f. Ohrenheilkunde. LVi. Bd. 
die Jugularis zu unterbinden ist, sind die Meinungen verschieden. v. Bergmann empfiehlt, wohl mit Recht, das Gefäss womöglich oberhalb der Einmündung der Vena facialis zu unterbinden, damit eine etwa rom oberen Bulbus abwärts steigende Thrombose nicht in diese Vene hineinwächst, wie es in der That von Littaur und Mensing beobachtet wurde."

Es ist richtig, dass v. Bergmann ("Ueber einige Fortschritte in der Hirnchirurgie". Arch. f. klin. Chir. Bd. 50 S. 321. 1895. Diese Literaturangabe findet sich bei Körner an dieser Stelle nicht) schon ein Jahr vor dem Erscheinen meiner Pyämiearbeit die Unterbindung der Jugularis oberhalb der Eintrittsstelle der Vena faciei communis empfohlen hat. Diese Arbeit war mir entgangen. In ihr sagt v. Bergmann, dass er die vorausgeschickte Unterbindung der Jugul. intern., "welche am besten und leichtesten oberhalb der Einmündung der Facialis communis ausgeführt wird", befürworte. Ich glaube, dass ein wesentlicher Unterschied in der Art besteht, in welcher v. Bergmann und ich die Unterbindung oberhalb der Gesichtsvene empfehlen. Ich habe meinem Vorschlage eine eingehende Begründung folgen lassen, unter Anderem auf die Gefahr des Fortschreitens der Thrombose auf die Vena facialis, die ich in meinem Falle VI vor Littaur und Mensing beobachtete, aufmerksam gemacht. Bei v. Bergmann findet sich diese Begründung meines Wissens erst in der III. Auflage seiner "Chirurgischen Behandlung der Hirnkrankheiten" des Jahres 1899.

Weiterhin darf ich annehmen, dass erst durch den von mir erfolgten Hinweis auf die Gefahren der Unterbindung unterhalb der Gesichtsvene, zu welchen insbesondere auch der Uebergang: infectiöser Stoffe von der Jugularis interna über die Vena facialis und über eine häufige Anastomose nach der Jugularis externa und in den Kreislauf gehört, der Vorschlag v. Bergmann's eine zwingende Begründung erfahren hat, nach welcher die Unterbindung unterhalb der Gesichtsvene in allen den Fällen als ein Kunstfehler angesehen werden muss, in welchem der Thrombus nicht tiefer in die Jugularis herabreicht. Eine derartige Bedeutung hat die Empfehlung v. Bergmann's, „weil sie am besten uad leichtesten ausführbar ist", jedenfalls nicht, was z. B. daraus hervorgeht, dass Dahlgreen (Penzoldt und Stinzing, Handbuch der speciellen Therapie der Erkrankungen des Bewegungsapparates und des Nervensystems u.s. w. Jena 1896. S. 1000) den v. Bergmann'schen Vorschlag verwirft. 